\title{
Políitica de Valorização do Salário Mínimo e sua Relação com o Desemprego
}

\section{Minimum Wage Valorization Policy and its Relation to Unemployment}

\author{
Rafael Luis Comini Curi ${ }^{\mathrm{a}}$ \\ Matheus Pereira Ribeiro ${ }^{b}$ \\ Cristiana Tristão Rodrigues ${ }^{\mathrm{C}}$
}

\begin{abstract}
Resumo: Este estudo busca identificar o vínculo existente entre um aumento do salário mínimo e a probabilidade de o indivíduo transitar de uma situação de empregado para a condição de desempregado, bem como pretende fornecer subsídio à formulação de políticas públicas. O período escolhido é de 2011 a 2015, após a nova lei de metodologia de reajuste do salário mínimo, e a análise de impacto realiza-se ano a ano, a partir de dados da Pesquisa Mensal do Emprego (PME). Para tanto, utilizam-se os métodos de propensity score matching e de diferença em diferenças. O grupo de tratamento é formado por indivíduos que recebem entre o antigo e o novo salário mínimo. Os indivíduos que recebem entre 1,5 e 2,5 vezes o novo salário mínimo, por sua vez, pertencem ao grupo de controle. Observa-se que, nas transições de 2011 para 2012 e de 2012 para 2013, o aumento do salário mínimo pode ter contribuído para o aumento da probabilidade de um indivíduo estar desempregado. Tal fato não é observado para os demais períodos analisados.
\end{abstract}

Palavras-chave: Salário mínimo. Emprego. Experimento natural. Políticas públicas.

Abstract: The study seeks to identify the link between an increase in minimum wage and the probability of the individual moving from an employment situation to an unemployed condition, as well as seeking to provide subsidy to the formulation of public policies. The period chosen was from 2011 to 2015, after a new minimum methodology readjustment law, and a one-year impact analysis based on data from the Monthly Employment Survey (PME). To do so, use the propensity score matching and differencein-difference methods. The treatment group in the form of issue receive the old and the new minimum wage. Those who want between 1.5 and 2.5 times the new minimum wage, in turn, belong to the control group. It was observed that, in the transition from 2011 to 2012 and 2012 to 2013 , the increase in the minimum wage may have contributed to the increase in the probability of an individual being unemployed. This fact was

a Fundação Getúlio Vargas (FGV), Escola de Economia de São Paulo (EESP), Departamento de Economia. São Paulo, São Paulo, Brasil.

b Universidade Federal de Juiz de Fora (UFJF), Departamento de Economia. Juiz de Fora, Minas Gerais, Brasil.

c Universidade Federal de Viçosa (UFV), Departamento de Economia. Viçosa, Minas Gerais, Brasil. 
not observed for the other periods analyzed.

Keywords: Minimum wage. Employment. Natural experiment. Public policy.

JEL Classification: E24; H53; J38.

\section{Introdução}

O presente estudo busca analisar o impacto da valorização do salário mínimo, como instrumento de política pública, no emprego nas regiões metropolitanas brasileiras. O período analisado será de 2011 a 2015, no qual ocorreu alteração na metodologia de valorização do salário mínimo, ganhando status de lei.

Em uma economia, a taxa de salários tem fundamental importância na explicação de fatores ligados à acumulação de capital e aos movimentos de variáveis ligadas à distribuição de renda. A taxa de salários consiste no salário-base pago à força de trabalho não qualificada por empresas que devem pagar o salário mínimo previsto em lei a seus trabalhadores. O salário mínimo, por sua vez, serve de guia para coordenar a política salarial do governo, de indicador do nível de preços e barganha salarial entre empregados e empregadores. Assim, seu efeito no emprego se dá de forma direta e indireta, por meio de salários, pensões, benefícios, inflação e déficit público. Fica clara, portanto, a importância do salário mínimo para a explicação de diversos fatores da dinâmica econômica de um país (SOUZA; BALTAR, 1979).

Alguns importantes trabalhos, com destaque para Foguel, Ulyssea e Corseuil (2014) e Pereira, Melo e Xavier (2017), entre outros, corroboram a teoria tradicional neoclássica de que, sob determinadas circunstâncias, o aumento do salário mínimo eleva o desemprego. Entretanto, trabalhos anteriores chegaram a conclusões distintas de ambos, como os estudos de Katz e Krueger (1992) e Corseuil e Carneiro (2001), que inferem que aumentos do salário mínimo afetam positivamente o emprego. Devido a essa controvérsia da literatura, faz-se necessário analisar a relação entre aumento do salário mínimo e desemprego, sobretudo em um contexto no qual se tem dado pouca atenção à robustez dos resultados encontrados, bem como aos critérios de estabilidade do modelo proposto. $\mathrm{O}$ estudo se torna ainda mais interessante ao se analisar essa relação após a implementação da nova metodologia de cálculo do salário mínimo.

Desse razoado, tem-se como contribuição a utilização de uma metodologia de "experimentos naturais", que captura um resultado causal por meio de um método quasi-experimental, pouco utilizada na análise do presente problema de pesquisa, principalmente para o caso brasileiro. O estudo ganha importância ao atualizar o período analisado, indo além ao utilizar testes para verificar a robustez 
e a estabilidade do modelo, como os testes de média, $R$ de Rubin e de grau de ajustamento.

\section{Revisão da Literatura}

Encontra-se na teoria neoclássica grandes contribuições acerca da relação entre mercado de trabalho e salários. A teoria afirma que o trabalho é remunerado pela sua respectiva produtividade marginal. De acordo com Soares (2002), tal fato pode originar, basicamente, quatro situações: primeiramente, na ausência de imperfeições entre produtividade e salário mínimo, não haveria desemprego. Em segundo lugar, com a inserção do salário mínimo, haveria desemprego dos trabalhadores que apresentassem produtividade menor que o valor do salário mínimo. Em terceiro lugar, com a possibilidade de desperdício de fatores, tal como o desemprego, as empresas poderiam se apropriar de uma parcela da produtividade marginal dos trabalhadores - ainda mais se estivessem desorganizados. Nesse contexto, o salário mínimo, além de proteger os trabalhadores dessa barganha, faria com que as empresas continuassem empregando e os trabalhadores procurassem trabalho com maior remuneração, o que levaria ao aumento do emprego. Por fim, o impacto do salário mínimo seria uma incógnita, com a possibilidade de aderência imperfeita do salário mínimo, tal como a presença da informalidade. Algumas empresas, além do poder de barganha, poderiam pagar menos do que o salário mínimo vigente. Por esse motivo, o aumento do salário mínimo não impactaria esse setor informal, mas poderia afetar o setor formal, posto que este deve seguir a legislação. Assim, o aumento do salário mínimo poderia acarretar mais custos para o setor formal, o que não necessariamente ocorreria no informal. Portanto, o resultado no desemprego dependeria de qual seria o maior resultado, se a diminuição do emprego no setor formal ou o aumento do emprego no informal. Tal caso se aproxima do contexto brasileiro, no qual a informalidade é significativa.

Em relação ao exposto pela teoria neoclássica em Soares (2002), Neri (1997) nos dá uma importante contribuição acerca do impacto dos salários mínimos sobre o desemprego. O autor, utilizando-se do conceito de efeito precarização, descreve o efeito do salário mínimo de expulsar indivíduos para fora do segmento formal do mercado de trabalho, ou seja, para uma situação de empregados sem carteira ou desocupados.

Foguel (1998) acrescenta outros dois importantes efeitos do salário mínimo: o efeito preço e o efeito farol. No primeiro, o aumento do salário mínimo leva ao aumento do custo de produção das firmas, dado o maior custo do trabalho não qualificado no setor formal. Logo, o aumento do salário mínimo pode levar ao aumento do emprego via setor informal ou à diminuição do emprego, via setor formal. O segundo é o papel de indexador para outros preços da economia. $\mathrm{O}$ 
aumento do salário mínimo pode ser uma referência para o ajuste salarial do setor informal, afetando o nível de salários e, consequentemente, o nível de emprego desse setor.

Sendo assim, não é possível afirmar de antemão qual será o efeito do salário mínimo sobre o emprego, ao menos teoricamente. A depender da magnitude do impacto do salário mínimo sobre os diversos setores de atividade econômica, é possível que a taxa de desemprego de um determinado setor aumente enquanto a de outro diminua.

A fim de validar as teorias aqui expostas, diversos trabalhos que analisam o impacto do salário mínimo sobre o mercado de trabalho tanto no Brasil quanto internacionalmente podem servir de apoio para este. Dessa forma, os resultados citados a seguir auxiliam na definição da melhor metodologia de análise a ser adotada, dado o problema de pesquisa proposto.

\subsection{Evidências Internacionais}

Katz e Krueger (1992) analisam o impacto de mudanças na lei de salário mínimo - que remunerava adolescentes abaixo do salário mínimo - na indústria de fast food nos Estados Unidos, entre dezembro de 1990 e agosto de 1991. As variáveis coletadas foram o número de empregados em tempo integral, o número de empregados em tempo parcial, o salário inicial médio para funcionários não gerentes e o aumento do salário mínimo federal. Para tanto, foram feitas duas regressões, uma para empresas que tiveram que aumentar muito o salário mínimo e outra para empresas que não foram tão afetadas, analisando variações no salário mínimo e o nível de emprego. Os autores utilizaram os métodos de mínimos quadrados ordinários (MQO) e de mínimos quadrados de dois estágios (MQ2E) e chegaram à conclusão de que o nível de emprego é positivamente correlacionado com variações no salário mínimo, contrariando a teoria tradicional.

Já Pereira (2005) busca analisar o impacto da lei que igualava o salário mínimo de jovens - que tinham menor valor salarial - aos de adultos no emprego em Portugal, entre 1986 e 1987. A autora utilizou um experimento natural, em que o grupo de tratamento são os jovens que obtiveram uma elevação de seus salários e o grupo de controle são os trabalhadores mais velhos que não foram impactados pela lei. O período analisado é de 1985 a 1989. Os resultados mostraram que o aumento do salário mínimo acarretou aumento do desemprego do grupo de tratamento em comparação ao grupo de controle. A autora conclui que o resultado era esperado, visto que o estudo se concentrou em trabalhadores jovens, cuja produtividade tende a ser menor. 


\subsection{Evidências Nacionais}

Ao analisar como o salário mínimo impacta a probabilidade de expulsão de trabalhadores do setor formal para o informal, Neri (1997) utiliza um experimento natural, traçando dois grupos de agentes: de tratamento e de controle. A conclusão é que a probabilidade de se deslocar de uma posição de trabalho formal para uma posição informal é mais que o dobro no grupo de tratamento, afetado pelo salário mínimo, em relação ao grupo de controle. O autor alerta, porém, sobre a probabilidade dessa transição a despeito da concessão ou não do reajuste do salário mínimo.

Foguel (1998), procurando estimar os efeitos do salário mínimo, utiliza o método diferença em diferença, analisando a convergência do salário mínimo nos estados brasileiros a partir de sua implementação, em 1984. Foi definido como grupo de tratamento os estados que estão convergindo para o salário mínimo maior - das regiões Norte, Nordeste e Centro-Oeste - e como grupo de controle os estados que não alteraram o valor do salário mínimo - Sul e Sudeste. O autor chega à conclusão que o aumento do salário mínimo elevaria tanto a taxa de desemprego agregada, bem como o desemprego dos que tinham trabalho anteriormente e também dos que não o possuíam.

Corseuil e Carneiro (2001) encontram, porém, uma forma mais precisa para analisar os impactos do salário mínimo sobre o mercado de trabalho brasileiro. Para tanto, os autores compararam os níveis de emprego observados antes da elevação do salário mínimo com níveis simulados referentes a algum momento após essa elevação, sem a interferência de nenhum outro fator além do aumento mencionado. Os resultados encontrados pelos autores revelaram que aumentos no salário mínimo tendem a promover a informalização do emprego. Tal fato seria compensado com outro resultado, no qual o emprego no setor formal tende a reagir de forma pró-cíclica a mudanças na atividade econômica, enquanto que o emprego informal reagiria de forma anticíclica a tais mudanças.

Trazendo considerável contribuição à literatura, Lemos (2004) estima os efeitos do salário mínimo sobre os níveis de emprego no Brasil no período de 1982 a 2000. A autora utiliza técnicas de dados em painel com dados sobre as famílias brasileiras em nível individual e regional. O resultado obtido informa que o salário mínimo afeta os trabalhadores, inicialmente, pelo número de horas por trabalhador, e não o número de empregos.

Procurando avaliar os efeitos da valorização do salário mínimo sobre as transições dos trabalhadores classificados como empregado (formal e informal) e empregado formal para classificação "informal, desempregado e inatividade", Foguel, Ulyssea e Corseuil (2014) utilizam dados da Pesquisa Mensal de Emprego (PME) do IBGE empregando o método diferença em diferença. Os autores seguem a meto- 
dologia proposta por Card e Krueger (1995) para definir os grupos de tratamento e controle. A análise é feita para cada episódio de aumento do salário mínimo desde 2003 até 2013. Para controlar as características dos indivíduos e, assim, determinar os dois grupos, os autores utiliza uma série de variáveis individuais, entre estas gênero, cor/raça, idade, se é chefe de família, escolaridade, entre outras, para controlar diferentes características observáveis entre os dois grupos. Os autores chegaram à conclusão de que aumentos do salário mínimo elevam a probabilidade de um empregado passar para o desemprego quando pertence ao grupo diretamente afetado no curto prazo, mas não no longo prazo. Da mesma forma, elevações do salário mínimo não parecem induzir um aumento das transições para o desemprego quando são considerados apenas os trabalhadores com carteira.

Por sua vez, Pereira, Melo e Xavier (2017) procuram analisar o impacto da política de salário mínimo no mercado de trabalho brasileiro no período de 2003 a 2015. Por meio dos dados da PME e utilizando o método de autorregressão vetorial (VAR), mostram que o salário mínimo levou ao aumento da taxa de desemprego. Os autores, porém, fazem análise em nível agregado, diferindo da literatura convencional.

Entende-se, até o momento, que o uso do método quasi-experimental se configura como metodologia relativamente conhecida nesse campo de análise. Percebe-se, no entanto, que não há consenso em relação ao efeito esperado da valorização do salário mínimo com relação ao emprego. Nesse sentido, este trabalho contribui para a literatura ao se preocupar com a robustez dos resultados encontrados e o balanceamento do modelo, representando, portanto, um avanço na fronteira do conhecimento, além de postular resultados empíricos novos e atuais para o presente problema de pesquisa.

\section{Breve Histórico do Salário Mínimo}

A noção do Estado como defensor dos interesses da classe trabalhadora no Brasil tomou forma ainda no período inicial do governo Getúlio Vargas (19301945). Esse novo momento do contexto político brasileiro - o fim da Primeira República (1889-1930) - demarca a formação da identidade do trabalhador brasileiro e dos salários (MERESSI, 2016).

O salário mínimo foi primeiro estipulado na Nova Zelândia, em 1894, e, em seguida, na Austrália, em 1896. Foi somente em 1934, porém, que o salário mínimo ingressou no ordenamento jurídico brasileiro, com a Constituição Federal. Até 1965, o salário mínimo aproximou-se muito de sua finalidade de proteção aos trabalhadores mais vulneráveis (MERESSI, 2016).

Durante o final dos anos 1970 e da primeira metade da década de 1980, observou-se um processo de convergência dos salários mínimos regionais no Brasil, visto que seu valor era diferente entre os estados. Essa diferença regional perdurou 
até 1984, ano em que ocorreu a unificação nacional do valor do salário (FOGUEL, 1998). A despeito dessa convergência, o período da ditadura militar pode ser caracterizado por grande perda do poder de compra do salário mínimo (MERESSI, 2016).

A partir de 2005, o ganho real do salário mínimo brasileiro passou a ser mais frequente (algo que já vinha sendo observado, embora em menor frequência, desde 1995). Na década seguinte, em 25 de fevereiro de 2011, foi editada a Lei $n^{\circ} 12.382$, que, além de fixar o valor do salário mínimo de 2011, estipulou a sua política de valorização de longo prazo. Essa regra estabeleceu que, no período compreendido entre 2012 e 2015, o índice de reajuste seria baseado na taxa de inflação (Índice Nacional de Preços ao Consumidor - INPC) do ano antecedente e o aumento real seria na magnitude da taxa de crescimento real do PIB de dois anos anteriores ao de vigência do novo salário mínimo. Assim, pode-se afirmar que, de 1995 a 2013, o salário mínimo experimentou uma trajetória de recomposição de seu valor real (MERESSI, 2016).

O período estudado - 2011 a 2015 - nos permite analisar a valorização real do salário mínimo sobre o desemprego após a implantação da Lei n ${ }^{\circ} 12.382$, que estipula como o salário mínimo passaria a se ajustar. Com o intuito de deixar clara a dinâmica do salário mínimo, seu comportamento será analisado nesta seção desde 1984.

O Gráfico 1 apresenta a evolução do salário mínimo real desde o momento de sua unificação. Antes de 1994, percebe-se que o valor do salário mínimo possuía oscilações frequentes e ausência de padrões.

\section{Gráfico 1 - Evolução do salário mínimo real}

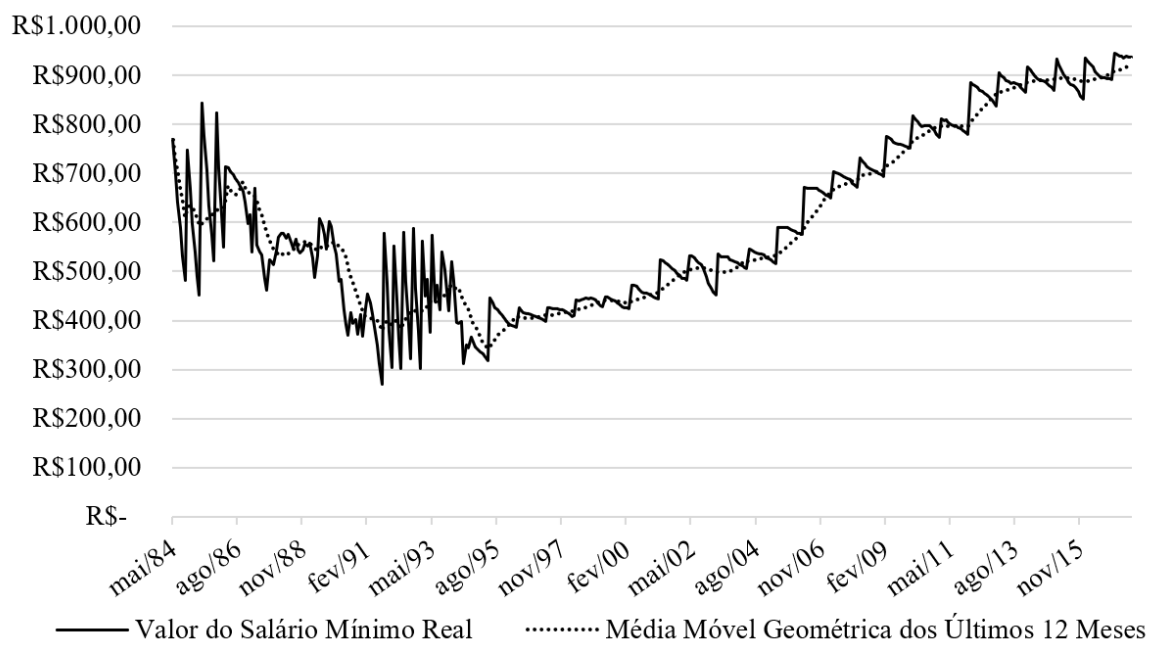

Fonte: Elaboração própria a partir de dados do Instituto de Pesquisa Econômica Aplicada (IPEA, 2019). 
De acordo com Foguel, Ulyssea e Corseuil (2014), nesse período, no qual crescentes taxas de inflação e constantes mudanças na política salarial eram praticamente cotidianas no contexto econômico brasileiro, a taxa de salários era utilizada como instrumento de estabilização macroeconômica. Entretanto, a partir de 1994, observou-se a recuperação do valor real do salário mínimo (em todos os anos, desde então a variação do salário mínimo foi superior à inflação, ou seja, sempre incorporando ganhos reais). ${ }^{1}$ Essa segunda fase pode ser dividida entre os diversos governos.

Durante o segundo mandato do governo Fernando Henrique Cardoso (1998-2002), o valor do salário mínimo real valorizou em média 4,35\%. Já durante o primeiro (2003-2006) e segundo mandato do governo Lula (2007-2010), aumentou em média $6,52 \%$ e $4,48 \%$, respectivamente. Durante o primeiro mandato da presidenta Dilma Rousseff (2011-2014), essa média cresceu em torno de 2,80\% e, em 2015, decresceu 0,8\%.

Após 2011, o valor do salário mínimo começa a ter ganhos reais cada vez menores. Isso pode ser atribuído ao fato da nova metodologia vigente, que vincula o crescimento da taxa de salários ao PIB real e a seus tímidos resultados no período.

Ao analisar os possíveis períodos de ganho do salário mínimo em busca de subsídio à afirmação anterior, pode-se dividir o período posterior a 1994 em três fases: a) de 1997 a 2004, quando a estabilização macroeconômica estava, de certa maneira, concretizada; b) de 2005 a 2010, no qual o salário inicia um período de maiores ganhos reais (em 2007, inicia-se o acordo para o ajuste da taxa de salários, que determina a reposição da inflação do ano anterior e o acréscimo do crescimento, se positivo, do PIB real de dois anos anteriores); e c) de 2011 a 2015, quando dá-se a oficialização da nova metodologia de valorização do salário mínimo até a sua reavaliação. Na primeira fase, o salário mínimo real cresceu em média 3,38\%. Já na segunda, cerca de 6,77\%. Finalmente, na terceira fase, esse crescimento foi de cerca de $2,07 \% .^{2}$

\section{Metodologia}

A fim de se identificar o impacto do aumento do salário mínimo na probabilidade de o indivíduo transitar de uma situação de empregado para a condição de desempregado, são utilizados métodos econométricos aplicados a políticas públicas. A metodologia proposta neste trabalho segue a mesma abordagem de Cor-

$1 \quad$ Esse cálculo foi feito a partir da variação do salário mínimo nominal do ano corrente em relação ao ano anterior comparada ao IPCA, o segundo índice calculado pelo Instituto Brasileiro de Geografia e Estatística (IBGE, 2018) e o primeiro retirado do Instituto de Pesquisa Econômica Aplicada (IPEA, 2019).

2 A média de ganhos reais durante os períodos foi calculada a partir da média da variação do valor real de julho do ano corrente em relação ao ano anterior durante os períodos pré-estabelecidos. 
seuil e Carneiro (2001) e Soares (2005), que reproduziram, por sua vez, o estudo canônico de Card e Krueger (1995). Especificamente, o método escolhido foi o diferenças em diferenças ponderadas pelo escore de propensão (double difference matching - DDM), que consiste na combinação das metodologias de pareamento por escore de propensão (propensity score matching - PSM) e diferença em diferenças (double difference - DD) para o caso não linear.

\subsection{Identificação}

Para a realização dos procedimentos aqui propostos, é necessário que se compare o grupo de tratamento em relação a um grupo de controle idêntico que não recebeu o tratamento (ou o mais próximo possível a isso) antes e depois do aumento do salário mínimo. Desse modo, procura-se reduzir os possíveis vieses de seleção, visto que se está comparando indivíduos semelhantes em determinadas características. A fim de controlar esses vieses, optou-se pela combinação do DD com o PSM, na qual aplica-se o DD na subamostra selecionada pelo PSM, visto que este realiza o pareamento de indivíduos tratados com indivíduos de controle.

Nesse caso, o PSM ajuda a minimizar os vieses provenientes da distribuição de características observáveis (gerado por diferenças na distribuição dos atributos observáveis entre tratados e controles) e de ausência de suporte comum (as amostras de tratados e controles podem não ter sobreposição da função de densidade condicional das características observáveis), enquanto que o DD reduz o viés de seleção por características não observáveis (decorre das diferenças em características não observáveis que influenciam conjuntamente o resultado e o recebimento do tratamento, condicionado às variáveis observáveis).

Com o intuito de dar continuidade a tal procedimento, delimitou-se, de maneira inicial, os grupos de tratamento (grupo afetado diretamente pela política de salário mínimo) e controle (grupo que possa ser comparável). O impacto do tratamento é dado pela diferença no resultado entre os dois grupos, ou seja, o que teria acontecido com o grupo de tratamento caso não houvesse o aumento do salário mínimo - o que leva ao conceito de contrafactual (FOGUEL, 1998). Tal delimitação será a mesma proposta por Foguel, Ulyssea e Corseuil (2014) e Soares (2005) e pode ser definida como segue.

O grupo de tratados será composto pelos indivíduos que tinham rendimentos entre o antigo e o novo valor do salário mínimo. A justificativa dessa simulação pode ser embasada na teoria neoclássica, na qual os indivíduos são remunerados pela sua respectiva produtividade do trabalho. É de se esperar que os indivíduos que recebem entre o antigo e o novo salário mínimo possuam uma produtividade naturalmente inferior em relação aos que recebem um salário maior. Logo, ao se aumentar o salário-base, supõe-se que tais indivíduos estarão sujeitos à tran- 
sição de condição de desocupados, dado que o novo salário é superior à sua produtividade.

O grupo de controle, por sua vez, inclui trabalhadores que recebiam entre 1,5 e 2,5 salários mínimos (do novo valor). A justificativa para tal intervalo é encontrada no trabalho de Soares (2005). Os valores limites 1,5 e 2,5 são múltiplos do salário mínimo e são escolhidos dada a hipótese de que trabalhadores que possuem salário indexado ao salário mínimo teriam uma produtividade semelhante à do grupo de tratados, mas com o salário ligeiramente superior, o que possibilita a comparação entre os grupos. Esse fato os deixaria sujeitos à transição para o desemprego. Neri (1997) também faz uso de intervalo semelhante (entre 1,5 e 3 salários mínimos) para o grupo de controle na definição do já mencionado efeito precarização. Já os trabalhadores que recebem acima desse intervalo possivelmente possuem uma produtividade maior do que a de trabalhadores que ganham o salário mínimo - mesmo que algumas características observáveis sejam semelhantes - e, assim, não seriam afetados pelo aumento.

É possível que a delimitação dos valores proposta não seja integralmente adequada para o procedimento realizado. Fatores como sazonalidade do emprego (festas de final de ano, por exemplo), ou demais eventos não relacionados com a política de valorização, podem influenciar a transição ou não para a condição de desocupado dos dois grupos, de maneira distinta. Isso invalidaria a hipótese da trajetória de evolução paralela na ausência da política de valorização. Essa possibilidade é apontada por Neri (1997), que sugere a comparação do reajuste em um intervalo maior de tempo, o que suavizaria as externalidades causadas pelos demais fatores não explicados pelo aumento do salário mínimo. ${ }^{3}$

Primeiramente, as características observáveis devem ser balanceadas, uma vez que a qualidade do pareamento depende delas para estimar a probabilidade de participação do grupo de tratamento. O segundo passo é estimar o índice de propensão associado ao grupo de tratados. Em seguida, calcula-se o suporte comum entre os dois grupos pelo PSM, que assegura, para cada indivíduo, a presença de um outro não tratado pareado, com valores similares em termos de escore de propensão - minimizando o viés de ausência de suporte comum. O método irá gerar a probabilidade condicional de um indivíduo fazer parte do grupo de tratamento com base em características observáveis. Por fim, um estimador do impacto da mudança de política é construído (SOARES, 2005).

Inicialmente, define-se $Y_{\text {it }}$ como o status de emprego observado para o indivíduo i. Já $Y_{i t}^{0}$ mostra se o indivíduo $i$, no tempo $t$, faz parte do grupo de controle. $Y_{i t}^{1}$

3 presente estudo procurou evitar essa possibilidade apontada por Neri (1997). Para isso, optou-se por realizar duas regressões distintas: uma para o curto prazo (novembro a fevereiro) e outra para o longo prazo (janeiro a abril dos anos anteriores ao aumento e janeiro a abril dos anos posteriores). No entanto, o segundo modelo não apresentou estabilidade. Assim, decidiu-se por trabalhar apenas com o curto prazo. 
é o indivíduo que pertence ao grupo de tratamento. $D_{i}$ é uma dummy de tratamento de determinado indivíduo $i$, à qual é atribuído o valor um se o indivíduo faz parte do grupo de tratados e zero, caso contrário. Por fim, $X_{\mathrm{it}}$ é um vetor de covariáveis explicativas observáveis (são fundamentais, pois está condicionada a elas a probabilidade de receber entre as faixas salariais e a estimação via DD) e $t$, o tempo ( $t=0$ é o momento antes do aumento do salário mínimo e $t=1$, após o aumento).

Ao se formalizar o PSM, tem-se o efeito médio do tratamento sobre os indivíduos tratados (average treatment effect on treated - ATT). Assumindo-se a hipótese de independência condicional (de forma que o pertencimento de determinado indivíduo ao grupo de tratados se torna aleatório e independe do tratamento), pode-se escrever o problema em termos de probabilidades. Após serem encontradas características observáveis que minimizem o viés de seleção (de forma que os indivíduos com características idênticas possuam a mesma probabilidade de serem alocados como tratamento ou controle), pode-se alcançar o parâmetro de interesse, definido como:

$$
\begin{aligned}
& \alpha=\left\{E\left[Y_{i t}^{1} \mid P\left(X_{i t}\right), D_{i}=1, t=1\right]-E\left[Y_{i t}^{1} \mid P\left(X_{i t}\right), D_{i}=1, t=0\right]\right\} \\
& -\left\{E\left[Y_{i t}^{0} \mid P\left(X_{i t}\right), D_{i}=0, t=1\right]-E\left[Y_{i t}^{0} \mid P\left(X_{i t}\right), D_{i}=0, t=0\right]\right\}
\end{aligned}
$$

em que $\alpha$ é o parâmetro de interesse que possibilitará o encontro de um grupo de indivíduos que sejam similares em todas as características observáveis $\left(X_{\mathrm{it}}\right)$ - que afetem a probabilidade do salário do indivíduo estar entre o antigo e o novo salário mínimo para encontrar um contrafatual. A título de exemplo, $E\left[Y_{i t}^{1} \mid P\left(X_{i t}\right), D_{i}=1, t=1\right]$ é a esperança de emprego no período $\mathrm{t}=1$, dado que o indivíduo faz parte do grupo de tratamento. Se dois indivíduos em grupos distintos (tratado e controle) possuem um escore de propensão semelhante, então considera-se que a probabilidade de o indivíduo receber entre o antigo e o novo salário mínimo é aleatória, fato que torna o grupo de controle válido.

Após determinar-se o grupo de controle o mais semelhante possível ao grupo de tratamento, utiliza-se o método $\mathrm{DD}$. O procedimento necessita de informação tanto do grupo controle quanto dos tratados em, pelo menos, dois períodos de tempo (antes e depois do aumento do salário mínimo). Tem-se por hipótese que, tendo-se controlado um conjunto de variáveis observáveis, caso a política de aumento do salário mínimo não tenha efeito, a trajetória dos resultados entre tratados e não tratados evoluiria paralelamente. Logo, o efeito do tratamento seria captado pela diferença dos resultados de antes e depois do aumento do salário mínimo. O escore de propensão pode ser usado para parear unidades de tratamento e controle no ano base (pré-tratamento), e, então, o impacto do tratamento é calculado sobre as unidades de tratamento e controle pareadas dentro do suporte comum. 


\subsection{Estimação}

Várias são as formas de se estimar esta probabilidade, a saber, $\mathrm{P}\left(x_{\mathrm{it}}\right)=\operatorname{Pr}$ $\left(\mathrm{Di}=1 \mid \mathrm{X}_{\mathrm{it}}=\mathrm{x}_{\mathrm{it}}\right)$. No presente caso, para se evitar que um indivíduo tratado em $\mathrm{t}+1$ seja pareado com um indivíduo em $t$, o que invalidaria o PSM, realiza-se o pareamento antes de cada aumento do salário mínimo. Escolheu-se o mês do novembro, e todos os indivíduos tratados entre dezembro e fevereiro teriam um par pareado em novembro. Ao se utilizar o escore de propensão a esses moldes, tem-se como hipótese que o comportamento dos dois indivíduos, no caso de ausência do tratamento, seria semelhante ao longo de todo o período. Essa hipótese é razoável, uma vez que a análise é feita para o curto prazo e as relações podem se manter constantes.

Deve-se ater, ainda, sobre a escolha a ser feita para o matching. No presente estudo utilizou-se o método probit. O algoritmo utilizado para o pareamento foi o do "vizinho mais próximo", no qual os indivíduos foram pareados com os que possuíam um escore de propensão próximo, ou seja, pares com características o mais semelhantes possível, e utilizado para a posterior estimação do DD. As variáveis são detalhadas na próxima subseção, e o modelo estimado para o PSM é dado por:

$$
\begin{gathered}
P\left(x_{i t}\right)=P_{r}\left(D_{i}=1 \mid X_{i t}\right)=\emptyset\left(\beta_{1}+\beta_{j} X_{i t}\right) ; j=(2,3,4,5,6,7) . \\
P\left(x_{i t}\right)=\emptyset\left(\beta_{1}+\beta_{2} \text { sexo }+\beta_{3} \text { cor }+\beta_{4} \text { De1a3AnosEst }+\beta_{5}\right. \text { De4a7AnosEst } \\
\left.+\beta_{6} \text { De8a10AnosEst }+\beta_{7} \text { Maisde11AnosEst }\right)
\end{gathered}
$$

no qual Ø (·) é a função de densidade do modelo probit; $D_{i}$ representa a variável binária (assume um se o indivíduo pertencer previamente ao grupo de tratados e zero se pertencer ao grupo de controle); o vetor $X_{i t}$ corresponde às características observáveis que influenciam o salário de um indivíduo e, logo, de pertencer ao grupo de tratados ou controle.

Já o estimador DD leva em consideração a inconsistência dos erros pela possibilidade de correlação serial. Ainda, há a possibilidade de os erros padrão dos coeficientes serem subestimados, fato que levaria à sobrerrejeição do efeito da política de salário mínimo e do viés de seleção. Tendo como hipóteses que o viés de seleção seja constante ao longo do tempo, ao se fazer a diferença da diferença, tal viés se anula. O estimador DD, de acordo com Blundell e Costa-Dias (2009), pode ser escrito como: ${ }^{4}$

4 Se a variável de interesse for uma dummy, a probabilidade poderá extrapolar o intervalo $[0,1]$. Blundell e Costa-Dias (2009) sugerem a utilização de um modelo probit para corrigir esse possível problema. Para a presente análise, verificou-se, por meio do software Stata 14.2, que, após a estimação de um modelo de probabilidade linear, ao se utilizar o comando predict (que salva as estimações para cada variável da amostra), tem-se que a probabilidade estimada não extrapolou o intervalo $[0,1]$ para nenhum dos anos estudados. Dessa forma, optou-se pela não utilização de um modelo probit. Em vez disso, utilizou-se um modelo MQO de efeitos aleatórios, seguindo-se a sugestão dos autores e apontado como o melhor modelo a ser utilizado ao se comparar efeitos 


$$
Y_{i t}=\beta+\alpha_{i} D_{i t}+u_{i t}
$$

em que

$$
E\left[u_{i t} \mid D_{i}, t\right]=E\left[n_{i} \mid D_{i}\right]+m_{t}
$$

Tem-se que $n$ é um efeito fixo individual não observado, $m$ é um choque macroeconômico agregado e $u_{i t}$ é o termo de erro aleatório. Pode-se simular o procedimento $\mathrm{DD}$ ao se realizar uma regressão de $Y_{\mathrm{it}}$ em $D_{\mathrm{it}}$ variáveis dummy para o grupo de tratamento, tempo, $t$, e (se necessário) outras variáveis explicativas de controle para se obter uma média de tratamento $\alpha$, ou seja, o impacto da política de salário mínimo no indivíduo tratado, no ponto médio da amostra. Para tanto, são feitas algumas suposições, como a homogeneidade de um efeito individual comum do tratamento após o programa, ou seja, um contrafatual que seja válido, e a de que a função de densidade de probabilidade segue uma distribuição normal. Ao fim, os autores mostram que é possível analisar a tendência apenas ao se comparar o resultado do grupo de controle antes e depois do aumento do salário mínimo somado ao contrafatual, formando um contrafatual não observado fornecido pelo PSM.

Dessa forma, o ATT, que determina o impacto da política de salário mínimo no grupo de tratados caso não houvesse o aumento de salário mínimo, pode ser utilizado para encontrar o parâmetro de interesse, que mede o impacto médio entre os tratados na inversa dos resultados esperados:

$$
\begin{gathered}
\alpha^{A T T}=E\left[\alpha_{i} \mid D_{i}=1\right] \\
=\left\{E\left[y_{i t} \mid D_{i}=1, t=1\right]-E\left[y_{i t} \mid D_{i}=1, t=t_{0}\right]\right\} \\
-\left\{E\left[y_{i t} \mid D_{i}=0, t=t_{1}\right]-E\left[y_{i t} \mid D_{i}=0, t=t_{0}\right]\right\}
\end{gathered}
$$

A equação 5 demonstra precisamente a estratégia de identificação do DD. Seu estimador, por sua vez, é representado como:

$$
\hat{\alpha}^{D D}=\left[\overline{y_{t_{1}}^{1}}-\overline{y_{t_{0}}^{1}}\right]-\left[\overline{y_{t_{1}}^{0}}-\overline{y_{t_{0}}^{0}}\right]
$$

em que $\overline{y_{t}^{D}}$ é a probabilidade média do grupo $D$ no tempo $t$. O DD mede o excesso de mudança na probabilidade para o grupo de tratados com relação ao grupo de controle, de forma a identificar o ATT: $E\left[\hat{\alpha}^{D D}\right]=\alpha^{A T T}$.

Nessa abordagem, o modelo de probabilidade linear estimado tem o seguinte formato: ${ }^{5}$

$$
Y_{i t}=\beta_{0}+\beta_{1} D_{i}+\beta_{2} T+\beta_{3} D_{i} T+u_{i t}
$$

fixos, aleatórios e pooled pelos testes de Breusch-Pagan, Hausman e Chow. 
no qual $Y$ representa a probabilidade de o trabalhador estar empregado no período $t$ (assume valor um se o indivíduo está ocupado e zero, caso contrário); $i$ indica o indivíduo; $\beta$ mostra o impacto médio de um indivíduo fazer parte do grupo de tratados em comparação ao grupo de controle na probabilidade de um indivíduo estar ocupado; $D_{i}$ assume um se o indivíduo pertence ao grupo de tratados e zero caso pertença ao grupo de controle; $\beta_{2}$ capta o impacto médio na variável dependente do ano posterior ao aumento em comparação ao ano anterior ao aumento, ou seja, um efeito fixo do tempo - assim, $T$ assume valor um se o período compreender a mudança no salário mínimo; $\beta_{3}$ mede o impacto médio, a partir da mudança no valor do salário mínimo, sobre a probabilidade de um indivíduo tratado estar ocupado, captando a interação entre as dummies de tratamento $\left(D_{i}\right)$ - valendo apenas a do mês de novembro - e de período (T); por fim, $u_{t i}$ representa o termo de erro aleatório.

\subsection{Base de Dados e Variáveis}

Inicialmente, para se avaliar o impacto de uma política, são necessárias informações sobre os indivíduos antes e depois dessa mesma política (FOGUEL, 2016). Como o salário mínimo tem aumentos todo ano, realiza-se uma análise ano a ano, de 2010 a 2011, de 2011 a 2012 e, assim, sucessivamente até 2015. O período antes da política será o primeiro ano e o período posterior à política será o ano final. Para o caso da análise do impacto do aumento do salário mínimo em 2011, o ano anterior à política será 2010, o posterior à política será 2011 e assim em diante.

O salário mínimo sofreu, no período de 2011 a 2015, alterações apenas no mês de janeiro, sempre no primeiro dia, segundo dados do Instituto de Pesquisa Econômica Aplicada (Ipea) (IPEA, 2019). Assim, será utilizado esse mês como o de aumento do salário mínimo.

Será utilizada a base de dados da Pesquisa Mensal de Emprego (PME) (IBGE, 2015). A pesquisa é realizada nas regiões metropolitanas de Porto Alegre, São Paulo, Rio de Janeiro, Belo Horizonte, Salvador e Recife, o que impede generalizações para o restante do país. Os domicílios são entrevistados durante quatro meses, ficando oito meses sem ser entrevistados e, após esse período, são acompanhados novamente por mais quatro meses. Foi trabalhado apenas com indivíduos que iniciaram as entrevistas em novembro do ano que antecede o aumento do salário mínimo e que finalizaram a pesquisa em fevereiro do ano seguinte. ${ }^{6}$

6 A PME produz indicadores mensais sobre a força de trabalho. Assim, é possível avaliar flutuações e tendências a médio e longo prazos de variáveis ligadas ao mercado de trabalho. A pesquisa abrange informações referentes à condição de atividade, condição ocupacional, rendimento médio nominal e real, posição na ocupação, posse de carteira de trabalho, entre outras variáveis, tendo como unidade de coleta os domicílios. Para mais detalhes sobre a estrutura da PME, ver Corseuil e Reis (2015). 
Como a PME não aloca nenhum código de identificação a cada indivíduo entrevistado, será utilizada a metodologia proposta por Ribas e Soares (2008), por meio do uso de um algoritmo de emparelhamento mais avançado do que o convencionalmente usado pelo IBGE. ${ }^{7}$

As variáveis utilizadas para calcular a probabilidade de um indivíduo fazer parte do grupo de tratados ao se utilizar o PSM estão de acordo com a literatura. ${ }^{8}$ Tais variáveis estão ligadas tanto a possível discriminação do mercado de trabalho quanto às características dos indivíduos, sendo elas: gênero, cor/raça e educação (escolheu-se como base de comparação para a escolaridade os indivíduos que possuíam menos de um ano de escolaridade ou eram analfabetos). Assim, as variáveis da equação 2 são definidas no Quadro 1:

Quadro 1 - Descrição das variáveis

\begin{tabular}{|l|l|}
\hline Variáveis & \multicolumn{1}{c|}{ Definição } \\
\hline Sexo & $\begin{array}{l}\text { Refere-se a uma dummy de sexo do indivíduo. É atribuído o valor um se do } \\
\text { sexo masculino e zero, caso contrário. }\end{array}$ \\
\hline Cor/raça & $\begin{array}{l}\text { Refere-se a uma dummy sobre a cor/raça do indivíduo. É atribuído o valor um } \\
\text { se o indivíduo é branco e zero, caso contrário. }\end{array}$ \\
\hline De1a3AnosEst & $\begin{array}{l}\text { Refere-se a uma dummy de escolaridade do indivíduo. Se o indivíduo possuir } \\
\text { de um a três anos de estudos, é atribuído o valor um; caso contrário, zero. }\end{array}$ \\
\hline De4a7AnosEst & $\begin{array}{l}\text { Refere-se a uma dummy de escolaridade do indivíduo. Se o indivíduo possuir } \\
\text { de quatro a sete anos de estudos, é atribuído um; caso contrário, zero. }\end{array}$ \\
\hline De8a10AnosEst & $\begin{array}{l}\text { Refere-se a uma dummy de escolaridade do indivíduo. Se o indivíduo possuir } \\
\text { de oito a dez anos de estudos, é atribuído um; caso contrário, zero. }\end{array}$ \\
\hline Maisde11AnosEst & $\begin{array}{l}\text { Refere-se a uma dummy de escolaridade do indivíduo. Se o indivíduo possuir } \\
\text { mais de 11 anos de estudos, é atribuído um; caso contrário, zero. }\end{array}$ \\
\hline
\end{tabular}

Fonte: Elaboração própria a partir de dados da PME (IBGE, 2015) tratados com o software Stata 14.2.

Como já mencionado, o grupo de tratados é definido como os trabalhadores que ganhavam entre o antigo e o novo salário mínimo e o grupo de controle refere-se aos trabalhadores que ganhavam entre 1,5 e 2,5 vezes o novo salário mínimo. Isso pode ser melhor visualizado na Tabela 1.

Tabela 1 - Definição dos grupos de tratamento e de controle

\begin{tabular}{lll}
\hline Período & Grupo de tratamento & Grupo de controle \\
\hline $2010-2011$ & $\mathrm{R} \$ 510 \leq$ SalarioPrinc $<\mathrm{R} \$ 540$ & $\mathrm{R} \$ 810 \leq$ SalarioPrinc $<\mathrm{R} \$ 1350$ \\
$2011-2012$ & $\mathrm{R} \$ 540 \leq$ SalarioPrinc $<\mathrm{R} \$ 622$ & $\mathrm{R} \$ 933 \leq$ SalarioPrinc $<\mathrm{R} \$ 1550$ \\
$2012-2013$ & $\mathrm{R} \$ 622 \leq$ SalarioPrinc $<\mathrm{R} \$ 678$ & $\mathrm{R} \$ 1017 \leq$ SalarioPrinc $<\mathrm{R} \$ 1695$ \\
\hline
\end{tabular}

Continuação...

$7 \quad$ Para mais detalhes sobre os problemas de identificação presentes na PME, ver Corseuil e Reis (2015) e Ribas e Soares (2008, 2010)

8 Ver Foguel (1998), Foguel, Ulyssea e Corseuil (2014), Soares (2005) e Arruda et al. (2009). 
conclusão...

\begin{tabular}{lll}
\hline Período & Grupo de tratamento & Grupo de controle \\
\hline \hline $2013-2014$ & $\mathrm{R} \$ 678 \leq$ SalarioPrinc $<\mathrm{R} \$ 724$ & $\mathrm{R} \$ 1086 \leq$ SalarioPrinc $<\mathrm{R} \$ 1810$ \\
$2014-2015$ & $\mathrm{R} \$ 724 \leq$ SalarioPrinc $<\mathrm{R} \$ 788$ & $\mathrm{R} \$ 1182 \leq$ SalarioPrinc $<\mathrm{R} \$ 1970$ \\
\hline
\end{tabular}

Fonte: Elaboração própria a partir de dados do Ipea (IPEA, 2019).

A variável SalarioPrinc é o salário do trabalho principal recebido pelo indivíduo na semana de referência. Dessa forma, foi possível traçar os grupos de tratamento e de controle de forma prévia para, posteriormente, analisar-se o suporte comum entre os grupos pelo PSM e, com a utilização do DD, calcular-se o impacto do aumento do salário mínimo, na transição desses anos, na probabilidade de um indivíduo estar ocupado.

\section{Resultados}

Nesta seção, serão apresentados os resultados do modelo descrito no capítulo anterior, ou seja, os impactos da valorização do salário mínimo na probabilidade de um indivíduo permanecer empregado para mudança anual do salário mínimo, de 2011 a 2015.

\subsection{Seleção do Grupo de Controle pelo Propensity Score Matching}

Mesmo estando os grupos de controle previamente definidos, somente foram utilizados efetivamente como grupo de controle os indivíduos que se situaram no suporte comum durante o processo de pareamento. Nesse processo, foi utilizado o método PSM com base nos cinco vizinhos mais próximos, de acordo com as características pessoais dos indivíduos. ${ }^{9}$

Os Gráficos 2 e 3 descrevem a média de cada variável por grupo de tratamento e de controle antes do pareamento. Observa-se, inicialmente, que há heterogeneidade entre os indivíduos em relação às características observadas nos dois grupos. Entende-se, portanto, que a utilização do PSM se faz necessária para minimizar o viés proveniente da diferença desses atributos.

$9 \quad$ As variáveis inseridas no modelo foram selecionadas por contribuírem para o balanceamento do modelo, sendo elas cor do indivíduo (assumindo um se o indivíduo for branco e zero, caso contrário), além do gênero (assumindo um se o indivíduo for do sexo masculino e zero se for do sexo feminino) e também dummies referentes à faixa de estudo atingida por um indivíduo - de um a três anos de estudo (um caso verdadeiro e zero caso contrário), de quatro a sete anos de estudo (um caso verdadeiro e zero caso contrário), de oito a dez anos de estudo (um caso verdadeiro e zero caso contrário) e mais de 11 anos de estudo (um caso verdadeiro e zero caso contrário). 
Gráfico 2 - Média dos indivíduos do grupo de tratados em relação às variáveis a serem utilizadas no PSM

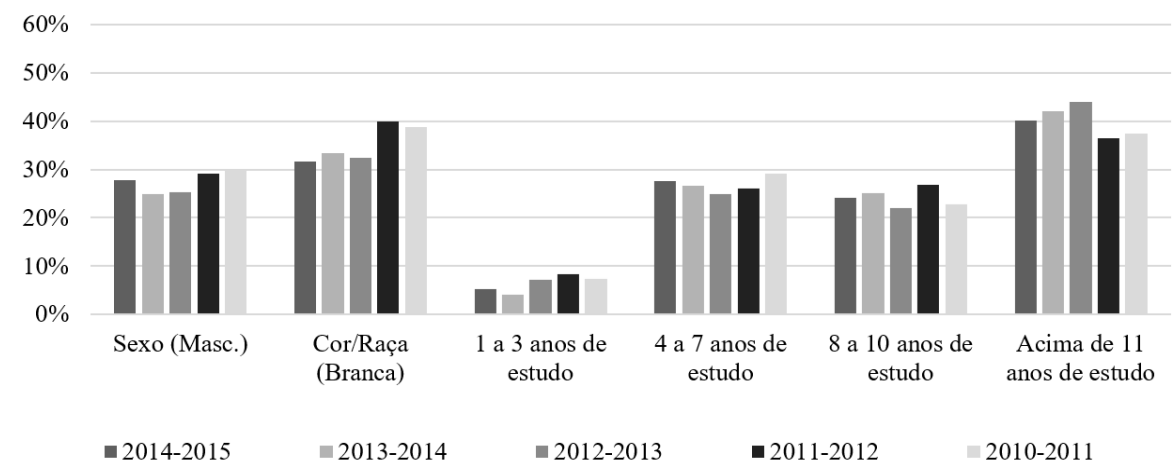

Fonte: Elaboração própria a partir de dados da PME (IBGE, 2015).

Gráfico 3 - Média dos indivíduos do grupo de controle em relação às variáveis a serem utilizadas no PSM

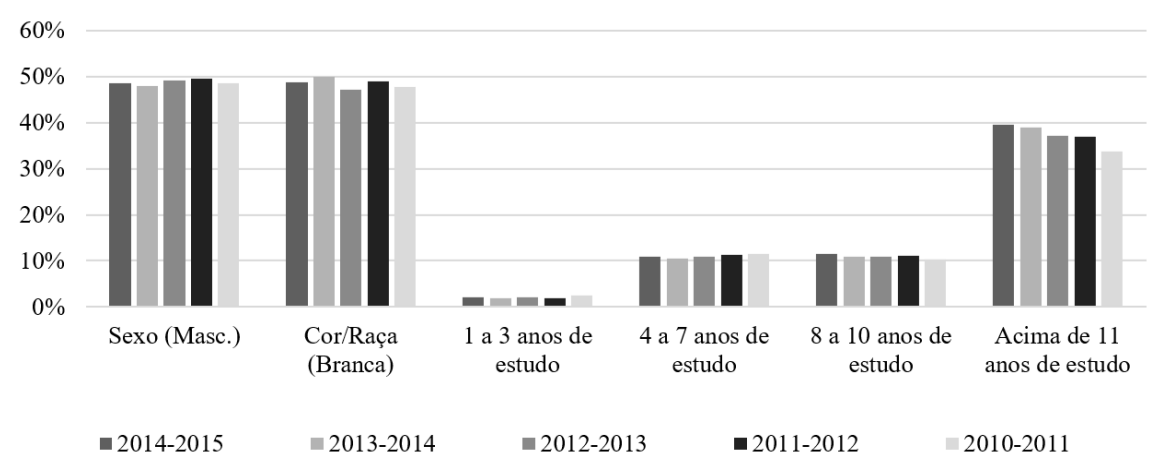

Fonte: Elaboração própria a partir de dados da PME (IBGE, 2015).

A probabilidade de um indivíduo fazer parte do grupo de tratamento para cada ano é apresentada na Tabela 2, presente no Apêndice A. As variáveis são analisadas no ano anterior ao aumento do salário mínimo, para cada ano. Elas buscam explicar a probabilidade de um indivíduo receber entre o antigo e o novo salário mínimo em comparação a indivíduos que recebem entre 1,5 e 2,5 vezes o novo salário mínimo. Nesse sentido, todas as variáveis explicativas mostraram-se estatisticamente significativas a no mínimo 5\% de significância para todos os anos.

Os resultados descritos na Tabela 2 evidenciam que, se o indivíduo for do sexo masculino, a probabilidade de fazer parte do grupo de tratamento é menor quando comparado às mulheres. $\mathrm{O}$ mesmo pode ser observado para indivíduos brancos. Tal fato sobressalta a discriminação racial e de gênero presente no merca- 
do de trabalho. A probabilidade de um indivíduo receber entre o antigo e o novo salário mínimo mostra-se menor à medida que o nível de escolaridade aumenta, o que evidencia a importância do capital humano na determinação do salário dos indivíduos. Essas observações são válidas para todos os anos analisados.

Além disso, verificou-se uma significância estatística conjunta das variáveis inseridas no modelo para todos os anos, fato que fica evidente por meio da estatística Likelihood Ratio (LR). Observou-se, ainda, que aproximadamente 15\% da variação no índice de tratamento é de fato explicada pelo modelo, como mostra o coeficiente de determinação (pseudoR ${ }^{2}$ ). Esse resultado é particularmente satisfatório, pois mostra que o modelo tem um bom poder explicativo, uma vez que há inúmeros fatores, além do aqui proposto, que possam explicar a remuneração de um indivíduo.

Após a realização do pareamento dos indivíduos por meio do PSM, foi realizado o teste de média entre os indivíduos do grupo de tratados e do grupo de controle, antes e após o pareamento para as variáveis explicativas utilizadas no PSM. Os resultados desse teste podem ser visualizados na Tabela 3, que verifica o percentual de redução de viés e sua significância. 


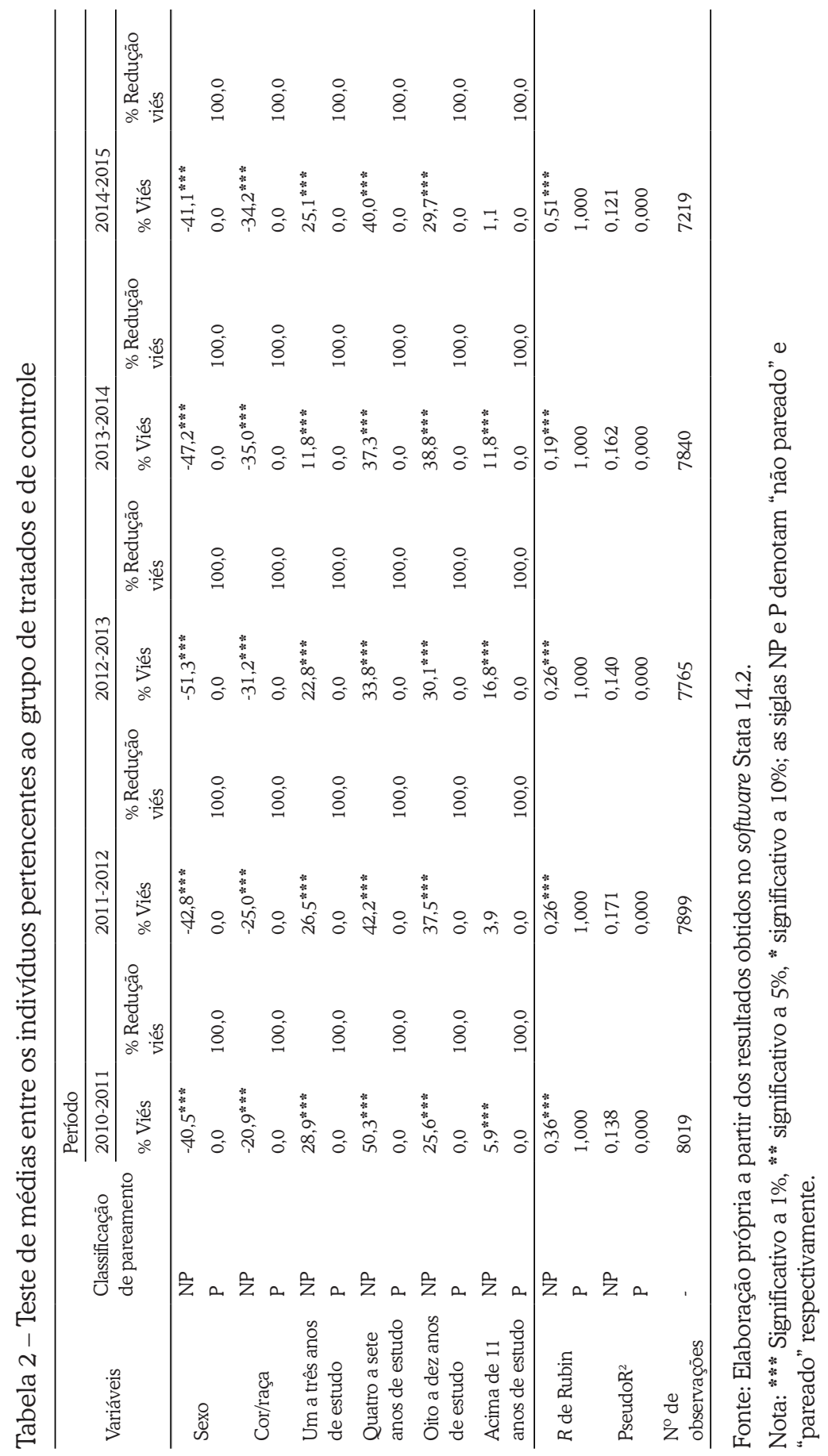


A partir dos resultados da Tabela 3, cuja hipótese nula é a igualdade do valor de cada variável para ambos os grupos - tratados e controle -, percebe-se que apenas para os anos de 2011 e 2015 (antes do pareamento) rejeita-se a hipótese nula da variável dummy de indivíduos que possuem mais de 11 anos de estudo. Portanto, as médias são significativamente diferentes. O restante das variáveis, mesmo antes do pareamento, ajudou a reduzir o viés entre as médias dos dois grupos. Após o pareamento, essa redução foi mais acentuada, uma vez que não se rejeitou a hipótese nula em nenhuma variável. Esse resultado mostra que a qualidade do pareamento foi satisfeita, ou seja, os dois grupos obtiveram características semelhantes.

A partir da estatística $R$ de Rubin, foi possível testar a hipótese de equilíbrio do modelo. Para todos os anos analisados, a propriedade de equilíbrio do modelo foi satisfeita, uma vez que os valores apresentados antes e após o pareamento situaram-se dentro dos limites de 0,5 e 2 respectivamente. Tal fato fornece subsídios para afirmar que a amostra é adequada para a análise.

Também foi testado o grau de ajustamento do modelo $^{10}$ ao se separar blocos de pareamento, a fim de verificar como cada variável contribui para o balanceamento do modelo nos respectivos blocos. A partir das variáveis explicativas inseridas no modelo, a propriedade de balanceamento foi satisfeita para todos os anos.

\subsection{Impacto do Aumento do Salário Mínimo sobre o Emprego}

Nesta seção, são apresentados os resultados do impacto do aumento do salário mínimo sobre probabilidade de o indivíduo transitar da condição de emprego para o desemprego, para todos os anos analisados após a nova metodologia de cálculo do salário mínimo. A estimação foi realizada de acordo com a equação (7), a partir de um modelo de probabilidade linear com a utilização de um MQO de efeitos aleatórios, com a proposta de um modelo DD não linear. Os resultados podem ser analisados a partir da Tabela 4.

Tabela 3 - Impacto do aumento do salário mínimo no emprego

\begin{tabular}{llllll}
\hline \multirow{2}{*}{ Variável } & Período & & & \\
\cline { 2 - 6 } & $2010-2011$ & $2011-2012$ & $2012-2013$ & $2013-2014$ & $2014-2015$ \\
\hline \multirow{2}{*}{ Tratamento } & $0,0934^{* * *}$ & $0,0939^{* * *}$ & $0,0954^{* * *}$ & $0,0716^{* * *}$ & $0,0866^{* * *}$ \\
& $(0,0188)$ & $(0,0143)$ & $(0,0177)$ & $(0,0145)$ & $(0,0186)$ \\
Período & $0,0163^{*}$ & $0,0150^{*}$ & $-0,0004$ & $-0,0005$ & $0,0155^{*}$ \\
Aumento do salário & $(0,0087)$ & $(0,0092)$ & $(0,0080)$ & $(0,0079)$ & $(0,0085)$ \\
mínimo & $-0,0201$ & $-0,0718^{* * *}$ & $-0,0583^{* * *}$ & $-0,0263$ & $-0,0309$ \\
\hline
\end{tabular}

Continuação...

10 O teste foi verificado a partir do comando pscore no software Stata 14.2. 
conclusão.

\begin{tabular}{llllll}
\hline \multirow{2}{*}{ Variável } & Período & & & \\
\cline { 2 - 6 } & $2010-2011$ & $2011-2012$ & $2012-2013$ & $2013-2014$ & $2014-2015$ \\
\hline Constante & $0,8969^{* * *}$ & $0,9006^{* * *}$ & $0,9003^{* * *}$ & $0,9194^{* * *}$ & $0,8989^{* * *}$ \\
Wald chi2 & $(0,0064)$ & $(0,0062)$ & $(0,0062)$ & $(0,0058)$ & $(0,0066)$ \\
Prob > chi2 & 27,37 & 46,35 & 32,77 & 24,98 & 23,72 \\
$\mathrm{R}^{2}$ (geral) & 0,0000 & 0,0000 & 0,0000 & 0,0000 & 0,0000 \\
$\mathrm{~N}^{\circ}$ de observações & 0,0109 & 0,0148 & 0,0108 & 0,0091 & 0,0095 \\
$\mathrm{~N}^{\circ}$ de grupos & 2995 & 3229 & 3279 & 3163 & 2843 \\
\hline
\end{tabular}

Fonte: Elaboração própria a partir de dados da PME (IBGE, 2015).

Nota: Os números sem parênteses são os coeficientes estimados, desvio padrão entre parênteses; $* * *$ significativo a $1 \%,{ }^{* *}$ significativo a $5 \%,{ }^{*}$ significativo a $10 \%$;

Ao se analisar o grupo de tratamento, constata-se que para todos os anos a variável foi estatisticamente significativa com 95\% de confiança. Se os indivíduos pertencerem ao grupo de tratados, a probabilidade de permanecerem empregados, a título de exemplo, aumenta em aproximadamente 9,34 pontos percentuais, na média, em comparação ao grupo de controle. Logo, os indivíduos que possuem um menor custo para a empresa, e que, portanto, se enquadram no grupo de tratamento, terão maior probabilidade de estarem empregados do que indivíduos presentes no grupo de controle, composto por aqueles que recebem entre 1,5 e 2,5 vezes o novo salário mínimo, porém com características similares ao grupo de tratamento. ${ }^{11}$ Foguel, Ulyssea e Corseuil (2014) também observam que os trabalhadores que recebem em torno do salário mínimo estão aumentando sua escolaridade de forma muito mais rápida que o restante da força de trabalho, fato que pode estar assegurando o emprego de tais trabalhadores frente ao grupo composto por pessoas pertencentes ao grupo de controle.

Com relação à dummy de período, observa-se que não foi possível rejeitar a hipótese nula ao nível de 5\% de significância em nenhum dos anos analisados. Sendo assim, os períodos após aumentos do salário mínimo não apresentaram efeito significativo em comparação aos anos anteriores ao aumento. É provável que o período analisado compreenda, de maneira teórica, o curto prazo. Se o período fosse relativamente maior, seria possível obter uma inferência sobre se de fato há possível antecipação dos agentes frente a aumentos do salário mínimo.

O impacto do aumento do valor do salário mínimo, por sua vez, foi estatisticamente significativo somente para dois anos, na transição de 2011 para 2012 e de 2012 para 2013. Nesses anos, respectivamente, o aumento do salário mínimo reduziu a probabilidade de o indivíduo estar empregado em aproximadamente 7,18 e 5,83 pontos percentuais respectivamente.

11 Vale notar que o pareamento foi realizado por meio do Propensity Score Matching (PSM). 
Para estes dois anos, o aumento do salário mínimo pode ter gerado demissões. Tal fato se embasa na teoria neoclássica, na qual os trabalhadores que possuem uma produtividade inferior ao aumento do salário mínimo seriam afetados pelo desemprego. De acordo com Foguel, Ulyssea e Corseuil (2014), esses efeitos adversos do salário mínimo podem ser justificados pelo fato do salário mínimo, além de representar um preço de referência para o mercado em geral, é especificamente relevante para certos segmentos da mão de obra. Assim, quando há aumento do salário mínimo de forma contínua e expressiva, sem uma contrapartida para o aumento da produtividade da mão de obra, é possível ocorrer expulsão dos indivíduos de seus postos de trabalho. Tal fato levaria o salário mínimo a ser ineficaz quanto à proteção social dos trabalhadores. Portanto, para esses dois anos tem-se por hipótese que o salário mínimo aumentou de maneira mais expressiva que a produtividade desses trabalhadores, especificamente nas regiões metropolitanas, de maneira a retirar trabalhadores do mercado de trabalho.

De forma a reduzir esse descompasso entre a evolução da produtividade do trabalho e a evolução do salário mínimo, Foguel, Ulyssea e Corseuil (2014) sugerem que a base de cálculo dos futuros reajustes do salário mínimo passasse a ser pautada por uma medida de produtividade do trabalho em vez do PIB. Como, no entanto, afirmam não haver dados que permitam esse cálculo, apontam que uma solução intermediária seria utilizar a produtividade média do trabalho na economia.

Para a transição dos anos 2010-2011, 2013-2014 e 2014-2015, o aumento do salário mínimo não parece induzir um aumento das transições para o desemprego. Uma explicação para a não observação das transições para o desemprego pode ser subsidiada por Soares (2002). O autor aponta para uma situação na qual, com a presença do setor informal na economia, o aumento do salário mínimo poderia levar a duas situações: o estímulo à formalização dos trabalhadores ou o estímulo a demissões por parte das firmas, devido aos custos mais elevados. Neste caso, o efeito final sobre o emprego dependeria do vetor resultante deste cenário, ou seja, um estímulo maior à formalização que supere o estímulo às demissões e vice-versa.

\section{Considerações Finais}

A relação existente entre salário mínimo e emprego ganhou destaque significativo para pesquisadores e especialistas em políticas públicas, sobretudo na última década, quando uma nova metodologia de cálculo ganhou status de lei e passou a definir um novo patamar para os ganhos salariais reais de toda a população. O presente estudo pretendeu, assim, identificar esse vínculo utilizando como ferramenta o PSM e o DD, no intuito de verificar se, no período posposto à nova metodologia de cálculo, houve efeito sobre o nível de emprego dos indivíduos. Para tanto, foram definidos o grupo de tratamento (indivíduos que recebem entre 
o antigo e o novo salário mínimo) e o de controle (indivíduos que recebem entre 1,5 e 2,5 vezes o novo salário mínimo).

Os resultados obtidos revelaram que, se o indivíduo for do sexo masculino, a probabilidade de fazer parte do grupo de tratamento é menor quando comparado às mulheres. $\mathrm{O}$ mesmo pode ser observado para indivíduos brancos. Ainda, notou-se que a probabilidade de um indivíduo receber entre o antigo e o novo salário mínimo mostra-se menor à medida que o nível de escolaridade aumenta.

Com relação ao impacto do salário mínimo sobre o emprego, observou-se que, na transição de 2011 para 2012 e de 2012 para 2013, o aumento do salário mínimo pode ter contribuído para o aumento da probabilidade de um indivíduo estar desempregado. Isso pode ser atribuído ao fato de o aumento da produtividade não acompanhar a valorização do salário mínimo. Nos demais períodos, observou-se a ausência desse impacto, de forma que o salário contribuiu como ferramenta de redução da desigualdade sem gerar desemprego. Assim, propõe-se uma nova metodologia de cálculo de reajuste do salário mínimo que inclua a produtividade média dos trabalhadores, fato que remuneraria os trabalhadores também pela sua produtividade, e não apenas de acordo com o crescimento do PIB. Dessa forma, seria possível evitar possíveis efeitos adversos do salário mínimo sobre o emprego no Brasil.

Esse resultado parece satisfatório no sentido de prover evidência empírica aos dois principais efeitos aqui esperados de um aumento no salário mínimo, qual sejam, aumento ou não do desemprego. Pode-se ainda sugerir, para os anos em que a valorização do salário mínimo contribuiu para a probabilidade de um indivíduo estar desempregado, que a sua aplicação enquanto política pública seja acompanhada de medidas que aumentem os ganhos de produtividade dos trabalhadores e trabalhadoras mais vulneráveis, ou seja, dos que ganham entre o antigo e o novo salário mínimo.

\section{Referências}

ARRUDA, E. F. et al. Determinantes da permanência no desemprego no mercado de trabalho cearense. In: ENCONTRO ECONOMIA DO CEARÁ EM DEBATE, 5, 2009, Fortaleza. Anais eletrônicos [...]. Fortaleza: IPECE, 2009. p. 161-177.

BLUNDELL, R.; COSTA-DIAS, M. Alternative approaches to evaluation in empirical microeconomics. Journal of Human Resourses, v. 44, n. 3, p. 565-640, 2009.

CARD, D.; KRUEGER, A. B. Mith and measurement: the new economics of the minimum wage. Princeton: Princeton University Press, 1995. 
CORSEUIL, C. H.; CARNEIRO, F. G. Os impactos do salário mínimo sobre emprego e salários no Brasil: evidências a partir de dados longitudinais e séries temporais. Texto para Discussão IPEA, n. 849, 2001.

CORSEUIL, C. H.; REIS, M. Há viés de painel rotativo na taxa de desemprego da PME? BMT IPEA, v. 58, p. 27-34, 2015.

FOGUEL, M. N. Uma avaliação dos efeitos do salário mínimo sobre o mercado de trabalho no Brasil. Texto para Discussão IPEA, n. 564, p. 1-31, 1998.

FOGUEL, M. N. Diferença-em-diferença. In: MENEZES FILHO, N. A. (ed.). Avaliação econômica de projetos sociais. 1. ed. São Paulo: Fundação Itaú Social, 2016. p. 99-125.

FOGUEL, M.; ULYSSEA, G.; CORSEUIL, C. H. Salário mínimo e mercado de trabalho no Brasil. In: CARDOSO JR., J. C. Brasil em desenvolvimento: estado, planejamento e políticas públicas. 1. ed. Brasília, 2014. v. 1, p. 295-323.

INSTITUTO BRASILEIRO DE GEOGRAFIA E ESTATÍSTICA (IBGE). Pesquisa Mensal de Emprego - PME. Rio de Janeiro: IBGE, 2015. Disponível em: < https:/www.ibge.gov.br/estatisticas/sociais/administracao-publica-e-participacao-politica/9183-pesquisa-mensal-de-emprego-antiga-metodologia.html?edicao=9545E $3 \mathrm{t}=$ microdados $>$. Acesso em: 01 nov. 2018.

INSTITUTO BRASILEIRO DE GEOGRAFIA E ESTATÍSTICA (IBGE). Índice Nacional de Preços ao Consumidor Amplo - IPCA. Rio de Janeiro: IBGE, 2018. Disponível em: https:// www.ibge.gov.br/estatisticas/sociais/administracao-publica-e-participacao-politica/9183-pesquisa-mensal-de-emprego-antiga-metodologia.html?edicao $=9545 \mathcal{E} \mathrm{t}=$ microdados. Acesso em: 01 nov. 2018.

INSTITUTO DE PESQUSA ECONÔMICA APLICADA (IPEA). Salário mínimo nominal vigente. Brasília: IPEA, 2019. Disponível em: http://www.ipeadata.gov.br. Acesso em: 01 nov. 2018.

KATZ, L. F.; KRUEGER, A. B. The effect of the minimum wage on the fast food industry. Industrial and Labor Relations Review, v. 46, n. 1, p. 6-21, 1992.

LEMOS, S. The effect of the minimum wage on prices the effect of the minimum wage on prices. Institute for the Study of Labor, n. 1072, 2004.

MERESSI, F. S. Salário mínimo e satisfação de necessidades vitais básicas no Brasil (20042013). 2016. Tese (Doutorado em Desenvolvimento, Sociedade e Cooperação Internacional) - Universidade de Brasília, 2016.

NERI, M. O reajuste do salário mínimo de maio de 1995. Boletim Conjuntural IPEA, n. 37, p. 49-51, 1997.

PEREIRA, S. C. The impact of minimum wages on youth employment in Portugal. Research Centre for Economic Policy: Research Memorandum, n. 4, p. 2-44, 2005.

PEREIRA, G. C.; MELO, A. S.; XAVIER, L. F. Efeitos da política de salário mínimo sobre o mercado de trabalho metropolitano: uma análise empírica a partir de vetores autorregressivos (Var) - (2003-2015). Revista de Economia Contemporânea, v. 21, n. 1, p. 1-23, 2017. 
RIBAS, R. P.; SOARES, S. S. D. Sobre o painel da pesquisa mensal de emprego (PME) do IBGE. Texto para Discussão IPEA, n. 1348, p. 7-38, 2008.

RIBAS, R. P.; SOARES, S. S. D. O atrito nas pesquisas longitudinais: o caso da pesquisa mensal de emprego (PME/IBGE). Estudos Econômicos, v. 40, n. 1, p. 213-244, 2010.

SOARES, S. O impacto distributivo do salário mínimo: a distribuição individual dos rendimentos do trabalho. Texto para Discussão IPEA, n. 873, 2002.

SOARES, F. V. Minimum wage hikes and employment transitions in Brazil. In:

ENCONTRO NACIONAL DE ECONOMIA, 33., 2005, Natal. Anais eletrônicos [...]. Natal: ANPEC, 2005.

SOUZA, P. R.; BALTAR, P. E. Salário mínimo e taxa de salários no Brasil. Pesquisa e Planejamento Econômico, IPEA/INPES, v.9, n.3,1979.

\section{Apêndice A - Resultados do PSM}

Tabela 4 - Resultados estatísticos do PSM com os cinco vizinhos mais próximos

\begin{tabular}{llllll}
\hline Variáveis & Período & & & & \\
\cline { 2 - 5 } & $2010-2011$ & $2011-2012$ & $2012-2013$ & $2013-2014$ & $2014-2015$ \\
\hline Intercepto & $-2,3733^{* * *}$ & $-2,2955^{* * *}$ & $-2,3337^{* * *}$ & $-2,4652^{* * *}$ & $-2,1481^{* * *}$ \\
& $(0,0932)$ & $(0,0950)$ & $(0,0546)$ & $(0,1208)$ & $(0,0876)$ \\
Sexo (masculino) & $-0,3611^{* * *}$ & $-0,4655^{* * *}$ & $-0.4774^{* * *}$ & $-0,5109^{* * *}$ & $-0,4047^{* * *}$ \\
& $(0,0547)$ & $(0,0462)$ & $(0,0534)$ & $(0,0510)$ & $(0,0552)$ \\
Cor/raça (branca) & $-0,1967^{* * *}$ & $-0,2769^{* * *}$ & $-0.2977^{* * *}$ & $-0,3613^{* * *}$ & $-0,2910^{* * *}$ \\
& $(0,0534)$ & $(0,0455)$ & $(0,1483)$ & $(0,0494)$ & $(0,0546)$ \\
Um a três anos de & $1,6067^{* * *}$ & $2,0170^{* * *}$ & $1,6273^{* * *}$ & $1,6493^{* * *}$ & $1,4918^{* * *}$ \\
estudo & $(0,1373)$ & $(0,1364)$ & $(0,1130)$ & $(0,1739)$ & $(0,1470)$ \\
Quatro a sete anos & $1,4393^{* * *}$ & $1,7357^{* * *}$ & $1,3807^{* * *}$ & $1,7793^{* * *}$ & $1,2624^{* * *}$ \\
de estudo & $(0,1031)$ & $(0,1039)$ & $(0,1139)$ & $(0,1309)$ & $(0,1016)$ \\
Oito a dez anos de & $1,2405^{* * *}$ & $1,7055^{* * *}$ & $1,3844^{* * *}$ & $1,7786^{* * *}$ & $1,1329^{* * *}$ \\
estudo & $(0,1081)$ & $(0,1048)$ & $(0,1050)$ & $(0,1304)$ & $(0,1021)$ \\
Acima de 11 anos de & $0,9873^{* * *}$ & $1,2904^{* * *}$ & $1,1410^{* * *}$ & $1,4184^{* * *}$ & $0,8241^{* * *}$ \\
estudo & $(0,0983)$ & $(0,0990)$ & $(0,1000)$ & $(0,1247)$ & $(0,0927)$ \\
LR chi2 & 431,32 & 806,69 & 459,86 & 649,65 & 363,54 \\
Prob > chi2 & 0,0000 & 0,0000 & 0,0000 & 0,0000 & 0,0000 \\
PseudoR & 0,1375 & 0,1706 & 0,1400 & 0,1625 & 0,1209 \\
o de observações & 8019 & 7899 & 7765 & 7840 & 7219 \\
\hline
\end{tabular}

Fonte: Elaboração própria a partir de dados da PME (IBGE, 2015).

Nota: Os números sem parênteses são os coeficientes estimados, desvio padrão entre parênteses; ${ }^{* * *}$ significativo a $1 \%,{ }^{* *}$ significativo a 5\%, ${ }^{*}$ significativo a $10 \%$. 


\section{Autor correspondente:}

Rafael Luis Comini Curi

Recebido em: 03/04/2018.

E-mail: rafaelccuri@gmail.com

Aceito em: 21/01/2019.

(cc) BY 